\title{
Efficacy and Tolerability of an mGlu2/3 Agonist in the Treatment of Generalized Anxiety Disorder
}

\author{
Eduardo Dunayevich*', Janelle Erickson', Louise Levine², Ronald Landbloom', Darryle D Schoepp ${ }^{2}$ and \\ Gary D Tollefson' \\ 'Orexigen Therapeutics, San Diego, CA, USA; ${ }^{2}$ Merck and Co., North Wales, PA, USA
}

\begin{abstract}
LY354740, a potent and selective mGlu (metabotropic glutamate receptor)2/3 agonist, has shown efficacy in the treatment of generalized anxiety disorder (GAD). LY544344 is a LY354740 prodrug that increases LY354740 bioavailability. This 8-week study was designed to evaluate the efficacy, safety, and tolerability of LY544344 in the treatment of GAD. Participants had a diagnoses of GAD, baseline Hospital Anxiety and Depression Scale anxiety subscale scores $\geqslant 10$, and moderate illness severity. Patients were randomized to double-blind treatment with LY544344 $16 \mathrm{mg}$ b.i.d. $(n=28)$, LY544344 8 mg b.i.d. $(n=36)$, or placebo $(n=44)$. LY544344 I6 mg b.i.d.treated patients showed significantly greater improvement from baseline in Hamilton Anxiety and Clinical Global Impression-Improvement scores, as well as response and remission rates compared with placebo-treated patients. LY544344 was well tolerated and there were no significant differences in the incidence of treatment-emergent adverse events among the three treatment groups. However, the trial was discontinued early based on findings of convulsions in preclinical studies. In conclusion, the findings of this study support the potential efficacy of $\mathrm{mGlu2/3}$ receptor agonist agents in the treatment of GAD. Additional studies will be needed to further assess the toxicological and clinical profile of LY354740/LY544344.

Neuropsychopharmacology (2008) 33, 1603-1610; doi:I0.1038/sj.npp. I301531; published online 22 August 2007
\end{abstract}

Keywords: GAD; LY354740; LY544344; mGlu2/3; glutamate

\section{INTRODUCTION}

Research in the past decade on glutamate receptors has provided a variety of new targets for pharmacological interventions. The family of metabotropic glutamate receptors (mGlu) comprises eight different receptor subtypes divided into three groups. These G-protein-linked receptors, which regulate production of second messengers and synaptic activity, control the release of multiple neurotransmitters and modulate neuronal excitability (Pin and Duvoisin, 1995; Schoepp, 2001). The mGlu2/3 receptors belong to the group II metabotropic receptor family and when expressed are coupled to inhibition of adenylate cyclase and modulation of ion channels. Functionally, activation of mGlu2/3 receptors in vivo suppresses enhanced synaptic excitability via pre- and post-synaptic mechanisms (Schoepp, 2001). These receptors are expressed primarily in forebrain regions, such as the amygdala and hippocampus, which are thought to play an important role in the expression of fear. Thus, they may also represent an important site of

*Correspondence: Dr E Dunayevich, Orexigen Therapeutics, 12481 High Bluff Drive, Suite 160, San Diego, CA 92130, USA, Tel: + I 858 436 8604, Fax: + I 858436 8650, E-mail: edunayevich@orexigen.com Received 5 March 2007; revised 10 July 2007; accepted 16 July 2007 action for anxiolytic drugs, including mGlu2/3 receptor agonists (Swanson et al, 2005).

LY354740, a glutamate analog, is a highly specific, nanomolar-potent agonist at mGlu2/3 receptors (Schoepp et al, 1997, 2003). LY354740 has evidenced activity in a number of rodent stress and anxiety models, including fear-potentiated startle, elevated plus maze, lactate-induced panic, and stress-induced hyperthermia (Walker et al, 2002; Tizzano et al, 2002; Monn et al, 1997; Shekhar and Keim, 2000; Spooren et al, 2002). Importantly, the effects of LY354740 occur at doses that are not associated with psychomotor impairment, which is common with benzodiazepine pharmacology (Helton et al, 1998; Tizzano et al, 2002). Data in transgenic knockout mice lacking mGlu2 or mGlu3 receptors have shown that the anxiolytic actions of LY354740 in the elevated plus maze test require the expression of one or both mGlu2/3 receptors (Linden et al, 2005).

In two human models of anxiety, fear-potentiated startle and panic induction by $\mathrm{CO}_{2}$-challenge, LY354740 showed significant effects in decreasing anxiety compared with placebo in healthy volunteers and panic disorder patients, respectively (Grillon et al, 2003; Schoepp et al, 2003). Anxiolytic activity was observed in initial, proof-of-concept studies in generalized anxiety disorder (GAD) (data on file, Eli Lilly and Co.) and in a large, multicenter, placebo and 
lorazepam-controlled GAD study (study LWAY; Michelson et al, 2005). In this study, 738 DSM-IV GAD patients were randomized to receive LY354740 administered as 100 or $200 \mathrm{mg}$ twice daily (b.i.d.), placebo or lorazepam $4-5 \mathrm{mg}$ daily, in a $3: 3: 3: 2$ ratio for up to 5 weeks of double-blind therapy. On the primary efficacy measure, the Hamilton Anxiety Scale (HAMA), both doses of LY354740 were associated with significantly greater efficacy than placebo and comparable to lorazepam $4-5 \mathrm{mg}$ daily with a superior tolerability profile. Treatment effect sizes (and 95\% confidence intervals (CIs)) were $0.29(0.09,0.48)$ and 0.21 $(0.01,0.41)$ for the LY354740 100 and $200 \mathrm{mg}$ b.i.d. groups, respectively; for the lorazepam $4-5 \mathrm{mg}$ daily group, it was $0.34(0.12,0.56)$. An exploratory analysis of the relationship between LY354740 plasma concentration and efficacy found an association between LY354740 plasma concentrations in the highest quartile and a higher rate of treatment response (defined as a decrease in HAMA total score from baseline $\geqslant 50 \%$ ).

The estimated oral bioavailability of LY354740 in humans is only $3-5 \%$ due to limited gastrointestinal absorption limiting potential therapeutic applications. To improve LY354740 absorption and further assess the therapeutic effects of mGlu2/3 agonists, a pharmacologically inactive peptidyl prodrug (L-alanine) form of the active compound LY354740, named LY544344, was developed. LY544344 is absorbed in the gastrointestinal tract through active PEP T1 transport, with eventual hydrolysis to produce molar equivalents of LY354740 and L-alanine (Bueno et al, 2005; Rorick-Kehn et al, 2006). In rodents, oral administration of LY544344 is associated with at least 10 -fold improvement in bioavailability compared with oral LY354740 and behavioral effects comparable to those seen with parenteral administration of LY354740 (Rorick-Kehn et al, 2006).

The findings reported in this paper are from the first efficacy study conducted with LY544344 in GAD. This study aimed to confirm that compounds that are agonists at the mGlu2/3 receptors can be associated with anxiolytic effects in clinical populations, as observed in previous studies with LY354740. The LY544344 doses selected for this study were expected to yield LY354740 plasma concentrations similar to those achieved in study LWAY (Michelson et al, 2005).

\section{MATERIALS AND METHODS}

\section{Study Design}

The study was a randomized, double-blind, parallel, placebo-controlled, fixed dose comparison of LY544344 $8 \mathrm{mg}$ b.i.d., LY544344 $16 \mathrm{mg}$ b.i.d., and placebo conducted at 23 sites in seven countries in western Europe and originally intended to randomize up to approximately 672 patients with a diagnosis of GAD. Ethical Review Boards provided written approval of the study protocol and written informed consent was obtained from each patient before study enrollment. This study was conducted in accordance with applicable laws and regulations, Good Clinical Practices, and the Declaration of Helsinki. The protocol specified that primary objective of this clinical study was to assess the efficacy of LY544344 compared with placebo in the treatment of GAD, as defined by the Diagnostic and Statistical Manual of Mental Disorders, Fourth Edition (DSM-IV). The primary efficacy assessment was the analysis of mean change from baseline to last observation of the HAMA total score (Hamilton Anxiety Rating Score; Hamilton, 1959) during the 8-week, double-blind acute treatment phase.

Patients who met inclusion and exclusion criteria were randomly assigned to one of three treatment groups: $8 \mathrm{mg}$ LY544344 b.i.d., $16 \mathrm{mg}$ LY544344 b.i.d., or placebo in a $1: 1: 1$ ratio. LY544344 or placebo was provided in identical opaque white capsules packaged in blister cards. To balance randomization groups for baseline anxiety severity levels, patients were stratified based on baseline HAMA scores $\leqslant 23$ or $>23$. Upon completion of the 8-week acute treatment phase or discontinuation of double-blind therapy, patients were requested to return for a follow-up visit to collect adverse event information 2 weeks after therapy discontinuation. Study drug was not tapered at the end of treatment.

Concomitant psychotropic medications were not allowed with the exception of zaleplon (up to $10 \mathrm{mg}$ ), zolpidem (up to $10 \mathrm{mg}$ ), zopiclone (up to $7.5 \mathrm{mg}$ ), or chloral hydrate (up to $1000 \mathrm{mg}$ ) at bedtime up to three times per week, if needed, for sleep. Patients receiving psychotherapy were permitted to enroll if therapy began at least 3 months before enrollment in the study, and neither introduction nor change in intensity or frequency of psychotherapy was permitted during the course of the study.

\section{Patient Population}

Patients had DSM-IV-defined GAD as assessed by a thorough psychiatric examination, including an interview with the Mini International Neuropsychiatric Interview (Sheehan et al, 1998) and review of medical records and collateral information, where available. Patients were men or women at least 18 years of age who presented at screening with a Hospital Anxiety and Depression Scale (HADS) (Zigmond and Snaith, 1983) anxiety subscale score of $\geqslant 10$, as well as a Clinical Global Impressions of Severity score $\geqslant 4$ (CGI-S; Guy, 1976), a Covi anxiety scale score $\geqslant 9$ (Lipman, 1982), and a Raskin depression scale score $\leqslant 7$ with no item $\geqslant 4$ (Raskin et al, 1969). No minimum HAMA score was required for inclusion into the study, to minimize any potential distortions in primary efficacy measure (HAMA) baseline scores (Moore et al, 2006). Patients with other current axis I disorders, with the exception of specific phobia, or lifetime history of psychotic disorders, bipolar disorder, obsessive-compulsive disorder, or factitious disorders were excluded. Patients were also excluded if their anxiety symptoms, in the investigator's opinion, were due to an underlying acute or chronic general medical condition or substance use/withdrawal, if they had an unstable serious illness (including cardiac, hepatic, renal, respiratory, endocrinologic, neurologic, or hematologic disease), or abnormal thyroid function, or had used a drug with psychotropic effects, other than the sedative hypnotics specified above, within 10 days before randomization. 


\section{Assessments}

The primary efficacy assessment used in this study was the HAMA total score. Other a priori efficacy assessments included the CGI-S; the Clinical Global ImpressionImprovement (CGI-I; Guy, 1976); the Montgomery Asberg Depression Rating Scale (MADRS; Montgomery and Asberg, 1979), and HADS. Treatment response was predefined as a CGI-I scale score of 1 (very much improved), or 2 (much improved), or a decrease from baseline in HAMA total score by at least $50 \%$. Remission was defined as a HAMA total score of 7 or less at the last visit in the acute treatment period (Ballenger, 2001). An investigator meeting was held before initiation of the study to instruct investigators and study raters on study procedures and review the use of relevant clinical rating scales. Prospective study raters were required to have previous clinical and research experience, to view a videotape of a clinical interview and provide HAMA individual item and total scores that were consistent with the scores provided by expert raters for that interview, and to conduct a brief mock interview in the presence of sponsor clinical personnel where items of the HAMA were rated. These procedures were conducted to ensure adequacy and consistency of clinical ratings across investigator sites.

Safety and tolerability were assessed via the collection of spontaneously elicited treatment-emergent adverse events at each visit, including serious adverse events and events leading to study discontinuation. Standard laboratory tests, including blood chemistry and hematology, were performed at screening and after 4 and 8 weeks of randomized therapy. Plasma concentration of LY544344 and LY354740 were assessed 2, 4, and 8 weeks after randomization, and electrocardiograms were performed at baseline and end point, or at the time of early discontinuation. Vital signs, including sitting blood pressure (systolic and diastolic), heart rate, and weight were also collected at all visits. A comprehensive physical examination was performed at screening and upon discontinuation of study drug treatment.

\section{Statistical Methods}

The a priori specified primary efficacy analysis was the comparison of LY544344 and placebo in mean change from baseline to last observation in the HAMA total score for the double-blind treatment phase. A linear combination of $\frac{1}{2}$ LY544344 $8 \mathrm{mg}$ b.i.d. $+\frac{1}{2}$ LY544344 $16 \mathrm{mg}$ b.i.d.-placebo was evaluated at the 0.05 significance level. The primary efficacy analysis was the comparison of LY544344 and placebo (ie, contrast hypothesis $=\frac{1}{2}$ LY544344 $8 \mathrm{mg}$ b.i.d. $+\frac{1}{2}$ LY544344 $16 \mathrm{mg}$ b.i.d. - placebo) in mean change from baseline to last observation in the HAMA total score during the doubleblind treatment phase. The linear combination was tested using a contrast hypothesis statement within the analysis of covariance (ANCOVA) model. The contrast compares the regression coefficients of the LY544344 treatment groups against placebo (Ho: $\left(\mathrm{B}_{\mathrm{LY} 16}+\mathrm{B}_{\mathrm{LY} 8}\right) / 2=\mathrm{B}_{\mathrm{P}}$ ). An ANCOVA model was used containing terms for treatment, investigator, and category of anxiety severity, with baseline HAMA total score as a covariate. If the primary hypothesis was statistically significant at the 0.05 , two-sided level, the LY544344 $8 \mathrm{mg}$ b.i.d. vs placebo and the LY544344 $16 \mathrm{mg}$ b.i.d. $v s$ placebo were tested at the 0.05 significance level without any penalty for multiple comparisons.

As this study was testing novel inclusion criteria where no minimum HAMA total score for enrollment into the study was required, to ensure that the patients included in the primary efficacy analysis would have adequate baseline severity a qualified population was prespecified: only those patients having a baseline HAMA total score $\geqslant 18$ and at least one post-baseline measure were included in the efficacy analyses. The criteria for qualification were blinded from investigators; patients not meeting these criteria were allowed to continue in the study. The study intended to randomize a total of approximately 672 patients. It was expected that approximately 185 patients per treatment arm would meet the a priori criteria for the qualified patient population. This sample size was expected to provide $80 \%$ power to detect a treatment effect size of 0.3 based upon a two-sided $0.05 t$-test. If the primary hypothesis was statistically significant at the 0.05 , two-sided level, pairwise comparisons with placebo were tested at the 0.05 significance level without penalty for multiple comparisons. Comparisons between treatment groups were assessed using an ANCOVA model containing terms for treatment, investigator, and baseline anxiety strata. The effect size for change in HAMA total score (a continuous variable) was created from Glass estimation (Hedges and Olkin, 1985). The effect size for change was calculated as the difference in mean change between the placebo group and the particular LY544344 dose group (a positive difference shows drug superiority) in the same study divided by the pooled sample standard deviation of the two treatment groups. Using the large sample asymptotic distribution property and under Ho, the effect size was asymptotically normally distributed with mean zero and the estimated variance of $\left(N_{\mathrm{d}}+N_{\mathrm{c}}\right) / N_{\mathrm{d}} N_{\mathrm{c}}+g_{2} / 2\left(N_{\mathrm{d}}+N_{\mathrm{c}}\right)$, where $N_{\mathrm{d}}$ and $N_{\mathrm{c}}$ denote the sample size for LY544344 and placebo treatment groups, respectively, and $g$ stands for the estimated effect size. An estimated $95 \%$ CI for the effect size was created utilizing these estimators and the critical values from a standard normal distribution. To further assess treatment effects over time, an observed case analysis of HAMA total score mean change from baseline to each visit using the model described above was also performed. CGI-I scores were analyzed using an analysis of variance (ANOVA) model containing terms for treatment, investigator, and baseline anxiety strata. Response and remission rates were analyzed using a Cochran-Mantel-Haenszel test for general association controlling for investigator and baseline anxiety strata.

All safety analyses were conducted on an intent-to-treat (ITT) basis. An ITT analysis is an analysis of data by the groups to which patients are assigned by random allocation, even if the patient does not take the assigned treatment, does not receive the correct treatment, or otherwise does not follow the protocol. Continuous safety variables were analyzed using an ANOVA model with treatment and investigator terms. Categorical safety variables were analyzed using Fisher's exact test. Treatment group differences were evaluated based on a two-sided, 0.05 significance level.

For the double-blind acute treatment phase analyses, baseline was defined as the last non-missing measurement before randomization. The last observation during the double-blind acute treatment phase was the last non-missing measurement obtained during the acute therapy phase. 
Table I Baseline Demographic and Clinical Characteristics ${ }^{\mathrm{a}}$

\begin{tabular}{|c|c|c|c|}
\hline \multirow{2}{*}{$\begin{array}{l}\text { LY544344 } \\
\text { b.i.d. }\end{array}$} & \multirow[b]{2}{*}{ Placebo } & \multicolumn{2}{|c|}{ LY544344 } \\
\hline & & $8 \mathrm{mg}$ b.i.d. & 16 mg b.i.d. \\
\hline Female, N (\%) & $27(61.4)$ & $18(48.6)$ & $15(50.0)$ \\
\hline Age, years, mean (SD) & $46.2(15.9)$ & $44.6(11.9)$ & $41.0(15.7)$ \\
\hline Caucasian, N (\%) & $44(100)$ & $36(97.3)$ & $29(96.7)$ \\
\hline Duration of illness, years (SD) & |3.4(|3.5) & $14.8(12.0)$ & $10.8(7.7)$ \\
\hline HAMA total score, mean (SD) & $27.00(5.05)$ & $26.72(4.84)$ & $28.6 \mid(4.88)$ \\
\hline $\begin{array}{l}\text { HADS anxiety subscale, } \\
\text { mean (SD) }\end{array}$ & $15.25(2.85)$ & $|4.6|(3.24)$ & $15.29(2.88)$ \\
\hline $\begin{array}{l}\text { HADS depression subscale, } \\
\text { mean (SD) }\end{array}$ & $6.89(3.51)$ & $7.22(3.34)$ & $6.79(3.32)$ \\
\hline MADRS total score, mean (SD) & $14.98(6.16)$ & $15.22(6.24)$ & |3.7| (4.26) \\
\hline CGI-S, mean (SD) & $4.66(0.68)$ & $4.83(0.70)$ & $4.93(0.72)$ \\
\hline
\end{tabular}

HAMA, Hamilton Anxiety Scale; HADS, Hospital Anxiety and Depression Scale; MADRS, Montgomery-Asberg Depression Rating Scale; CGI-S, Clinical Global Impression Scale, Severity.

${ }^{a}$ Demographic characteristics are provided for the ITT (intent-to-treat) population $(N=|| \mid)$, baseline clinical characteristics correspond to the qualified subset $(N=108)$ (placebo: $N=44$, 44; LY544344 $8 \mathrm{mg}$ b.i.d.: $N=37,36$; LY544344 $16 \mathrm{mg}$ b.i.d.: $N=30$, 28, respectively, for ITT and qualified groups). No statistically significant differences between treatment groups were observed.

\section{RESULTS}

\section{Patient Demographics and Disposition}

Owing to findings of convulsions in preclinical studies with LY544344, the study was stopped and active patients were discontinued before the full prespecified sample $(\sim 672$ patients) was enrolled. At the time of study discontinuation, 148 patients had provided informed consent, with 111 patients randomized. Of $108(97.3 \%)$ patients meeting criteria for qualified patients, $103(92.8 \%)$ had baseline and at least one post-baseline observation. Sixty-eight patients were discontinued due to premature study termination, six due to adverse events, six due to lack of efficacy, and two due to lost to follow-up; there were no meaningful differences in patient disposition across treatment groups. Only 29 patients (26.1\%) completed the double-blind treatment phase and entered the single-blind placebo, drug discontinuation assessment phase. Clinical and demographic characteristics of the study sample are presented in Table 1. Number of patients remaining in the study by treatment and week of therapy are provided in Figure 1; there were no significant differences among treatment groups on baseline characteristics or disposition.

\section{Efficacy Results}

The efficacy results are based upon the qualified subset; results for the entire randomized sample did not differ significantly from the results in the qualified subset. Results of the primary efficacy analysis indicated a positive treatment effect for patients in the LY544344 treatment groups relative to placebo treatment $(p=0.027)$. Patients who received LY544344 $16 \mathrm{mg}$ b.i.d. showed significantly greater mean improvement from baseline on the HAMA total score than patients who received placebo (Figure 1).

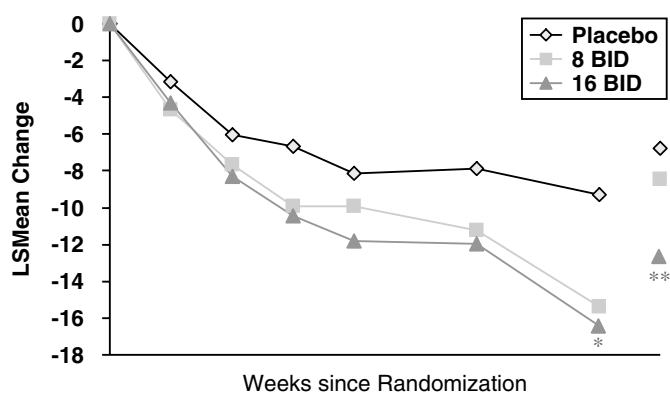

$\begin{array}{lcccccccc} & \mathbf{0} & \mathbf{1} & \mathbf{2} & \mathbf{3} & \mathbf{4} & \mathbf{6} & \mathbf{8} \text { Endpoint } \\ \begin{array}{llllllll}\text { N remaining by week: } \\ \text { 16 mg BID }\end{array} & 28 & 26 & 24 & 22 & 20 & 19 & 11 & 26 \\ 8 \mathrm{mg} \mathrm{BID} & 36 & 35 & 32 & 26 & 20 & 18 & 11 & 35 \\ \text { Placebo } & 44 & 42 & 36 & 32 & 28 & 22 & 16 & 42\end{array}$

${ }^{*} \mathrm{P}<0.05,{ }^{* *} \mathrm{P}<0.01$

Figure I Mean HAMA total scores by visit (observed cases) and end point (week 8, LOCF), qualified patients, double-blind treatment phase.

While improvement on the HAMA total score between the LY544344 $16 \mathrm{mg}$ b.i.d. and $8 \mathrm{mg}$ b.i.d. treatment groups was not statistically different, the $8 \mathrm{mg}$ b.i.d. dose group failed to separate from placebo. Effect size (based upon HAMA total score) for the $16 \mathrm{mg}$ b.i.d. treatment group was 0.84 $(95 \%$ CI: $0.33,1.35)$ and for the $8 \mathrm{mg}$ b.i.d. treatment group was 0.20 (95\% CI: $-0.25,0.65)$. Secondary mixed-model repeated measures analyses of both the ITT and the qualified subset also indicated significantly greater efficacy for LY544344 $16 \mathrm{mg}$ b.i.d. but not for LY544344 $8 \mathrm{mg}$ b.i.d. therapy compared with placebo therapy on the HAMA total score.

In the observed case analysis of the HAMA total score, the LY544344 $16 \mathrm{mg}$ b.i.d.-treated groups experienced a significantly greater improvement from baseline than the placebo-treated patients at week 8 (Figure 1). The LY544344 $8 \mathrm{mg}$ b.i.d. treatment group showed numerically greater mean improvement than the placebo treatment group at week 8 , but this difference was also not statistically significant (Figure 1).

The LY544344 $16 \mathrm{mg}$ b.i.d. treatment group demonstrated significantly greater improvement from baseline than the placebo treatment group on the HAMA psychic anxiety factor $(p=0.019)$ and the somatic anxiety factor scores $(p=0.005)$ (Figure 2). Response rates were significantly greater for the LY544344 $16 \mathrm{mg}$ b.i.d. group than the placebo group $(N=14,54 \%$ vs $N=10,24 \%$; $p=0.008)$. LY5 54344 $8 \mathrm{mg}$ b.i.d. response rates were numerically but not statistically superior to placebo $(N=13,37 \%)$. Remission rates were significantly different for both LY groups compared with placebo ( $16 \mathrm{mg}$ b.i.d.: $N=5,19 \% ; 8 \mathrm{mg}$ b.i.d.: $N=7$, 20\%; placebo: $N=0,0 \% ; p=0.012$ and 0.023 , respectively).

Secondary efficacy measures, including the HADS anxiety and depression subscale scores, MADRS total score, and CGI-S, failed to demonstrate a significant difference in mean change from baseline for any treatment group. CGI-I scores indicated significantly more improvement for the LY544344 $16 \mathrm{mg}$ b.i.d. group than both LY544344 $8 \mathrm{mg}$ b.i.d.- and placebo-treated groups (CGI-I scores: $16 \mathrm{mg}$ b.i.d., 2.56; $8 \mathrm{mg}$ b.i.d., 3.30; placebo, 3.15; $p=0.012$ and 0.037 , respectively). There were no significant differences between LY544344 $8 \mathrm{mg}$ b.i.d. and placebo on the CGI-I. 


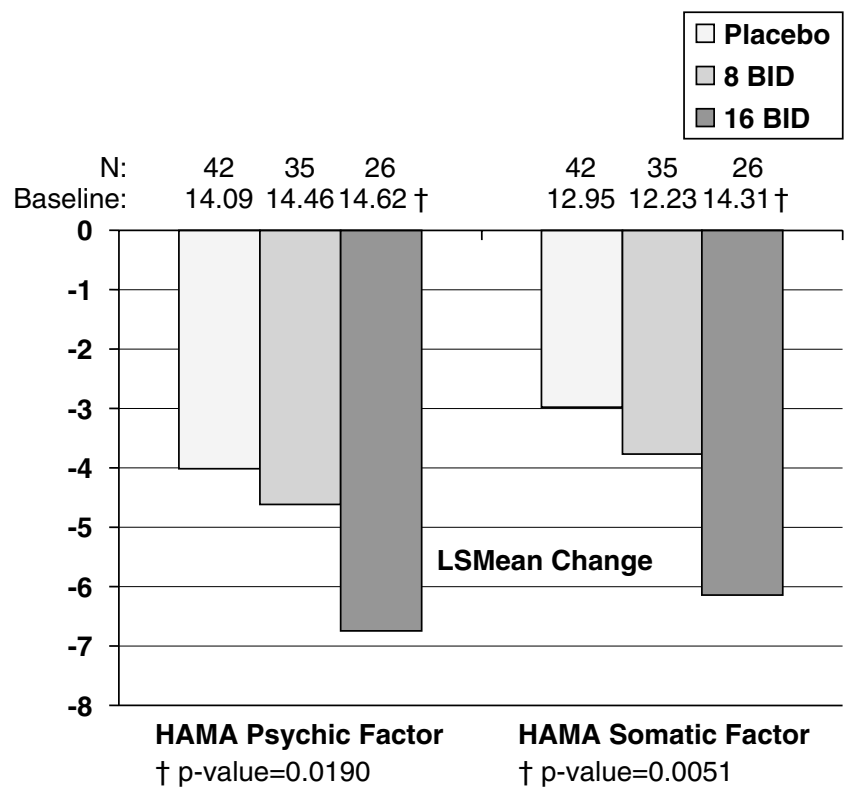

Figure 2 Mean HAMA psychic and somatic factor scores, least-squares mean change, baseline to last observation, qualified patients, double-blind treatment phase.

Table 2 Ten Most Commonly Reported Treatment-Emergent Adverse Events $^{\mathrm{a}}$

\begin{tabular}{|c|c|c|c|c|}
\hline Event & $\begin{array}{c}\text { Placebo } \\
\begin{array}{c}N=44 \\
n(\%)\end{array}\end{array}$ & $\begin{array}{c}8 \text { mg b.i.d. } \\
\begin{array}{c}N=37 \\
n(\%)\end{array}\end{array}$ & $\begin{array}{c}16 \text { mg b.i.d. } \\
\begin{array}{c}N=30 \\
n(\%)\end{array}\end{array}$ & $\begin{array}{c}\text { Total } \\
\text { N= I I I } \\
\text { n (\%) }\end{array}$ \\
\hline Patients with $\geqslant 1$ TEAE & $25(56.8)$ & $20(54.1)$ & $20(66.7)$ & $65(58.6)$ \\
\hline Nausea & $5(11.4)$ & $8(21.6)$ & $9(30.0)$ & $22(19.8)$ \\
\hline Headache & $2(4.5)$ & $3(8.1)$ & $3(10.0)$ & $8(7.2)$ \\
\hline Abdominal pain (upper) & $2(4.5)$ & $2(5.4)$ & I (3.3) & $5(4.5)$ \\
\hline Dizziness & $2(4.5)$ & $2(5.4)$ & $2(6.7)$ & $5(4.5)$ \\
\hline Fatigue & $2(4.5)$ & I (2.7) & I (3.3) & $5(4.5)$ \\
\hline Diarrhea NOS & $2(4.5)$ & $2(5.4)$ & $2(6.7)$ & $4(3.6)$ \\
\hline Dysmenorrhea & $2(4.5)$ & $0(0.0)$ & $2(6.7)$ & $4(3.6)$ \\
\hline Dyspepsia & $2(4.5)$ & $0(0.0)$ & I (3.3) & $3(2.7)$ \\
\hline Feeling abnormal & $2(4.5)$ & I (2.7) & $0(0.0)$ & $3(2.7)$ \\
\hline Insomnia & $3(6.8)$ & $0(0.0)$ & $0(0.0)$ & $3(2.7)$ \\
\hline
\end{tabular}

TEAE, treatment-emergent adverse events.

${ }^{a}$ There were no significant differences in the incidence of adverse events across treatment groups.

\section{Adverse Events}

The most frequently reported treatment-emergent adverse event among randomized patients in all three treatment groups was nausea (Table 2). This event was reported by 30 , 21 , and $11 \%$ of patients receiving LY544344 $16 \mathrm{mg}$ b.i.d., LY544344 $8 \mathrm{mg}$ b.i.d., and placebo, respectively; this difference was not statistically significant. Headache also was reported by at least $5 \%$ of patients who received LY544344 $8 \mathrm{mg}$ b.i.d. and $16 \mathrm{mg}$ b.i.d. and more frequently than in patients receiving placebo, but these differences were also not significant. Other treatment-emergent adverse event rates were generally comparable among the treatment groups. There were no deaths or other serious adverse events reported during this study. During the double-blind treatment phase, one patient who received LY544344 $8 \mathrm{mg}$ b.i.d., two patients who received LY544344 $16 \mathrm{mg}$ b.i.d., and three patients who received placebo discontinued the study because of adverse events. Evaluation of laboratory analytes, vital signs, and ECG results did not reveal any clinically meaningful changes among patients treated with LY544344 compared with patients treated with placebo.

\section{LY354740 Plasma Concentrations}

Plasma samples for determination of LY544344 and LY354740 plasma concentrations were obtained approximately 2, 4, and 8 weeks after initiation of randomized treatment (Table 3). LY354740 mean plasma concentrations observed after administration of LY544344 8 or $16 \mathrm{mg}$ b.i.d. were numerically higher, but generally within the range of LY354740 levels observed in previous clinical studies using LY354740 100 or $200 \mathrm{mg}$ b.i.d. (mean concentrations (SD) after 5 weeks of therapy, study LWAY: $67.8(37.5) \mathrm{ng} / \mathrm{ml}$ and $126(85.3) \mathrm{ng} / \mathrm{ml}$, respectively, data on file, Eli Lilly and Co.).

\section{DISCUSSION}

The findings of this multicenter, randomized, double-blind, placebo-controlled clinical study of LY544344 8 or $16 \mathrm{mg}$ b.i.d. are consistent with the preclinical and clinical data reporting anxiolytic effects with the administration of mGlu2/3 agonist agents. In spite of limited statistical power, LY544344 $16 \mathrm{mg}$ b.i.d. demonstrated a statistically significant reduction on the HAMA total score and psychic and somatic subscales relative to placebo and corresponding efficacy as determined by the CGI-I and response and remission rates. Compared to the results observed in LWAY, the more robust effects of treatment at the high LY544344 dose may have been partly because of the improved bioavailability of LY354740 offered by LY544344, as well as innovations in protocol design. It is important to note, however, that the small sample size and large number of discontinuations not at random due to premature trial closure may increase the likelihood of a type I error and bias in the interpretation of these findings. For example, the lowest proportion of patients completing acute therapy occurred in the LY544344 $8 \mathrm{mg}$ b.i.d. group $(N=11,31 \%)$, the highest occurred in the $16 \mathrm{mg}$ b.i.d. group $(N=11$, $42 \%)$, with intermediate completion rates $(N=16,38 \%)$ in the placebo group. This could have increased the likelihood of a more favorable result for the LY544344 $16 \mathrm{mg}$ b.i.d. group. The numerically shorter duration of illness in the LY544344 $16 \mathrm{mg}$ b.i.d. group may also have led to more favorable outcomes in that group. Unlike in study LWAY (Michelson et al, 2005 and data on file, Eli Lilly and Co.), the LY544344 lower dose (8 $\mathrm{mg}$ b.i.d., approximately equivalent to LY354740 $100 \mathrm{mg}$ b.i.d.) showed limited evidence of efficacy, and no statistically significant treatment effects were observed for the LY544344 $16 \mathrm{mg}$ b.i.d. group at any time point before week 8 . In this study, there were also no 
Table 3 Plasma Concentrations of LY354740 in GAD Patients after 8 and 16 mg b.i.d. Administration of LY544344

LY544344 8 mg b.i.d.

\begin{tabular}{|c|c|c|c|c|c|}
\hline \multirow[b]{2}{*}{ Variable } & \multirow[b]{2}{*}{ Statistic } & & & & \\
\hline & & Visit 4 & Visit 6 & Visit 8 & Overall \\
\hline & CV (\%) & 61.3 & 88.5 & 65.9 & 75.1 \\
\hline & Median & 83.23 & 47.91 & 48.76 & 54.76 \\
\hline
\end{tabular}

LY544344 16 mg b.i.d.

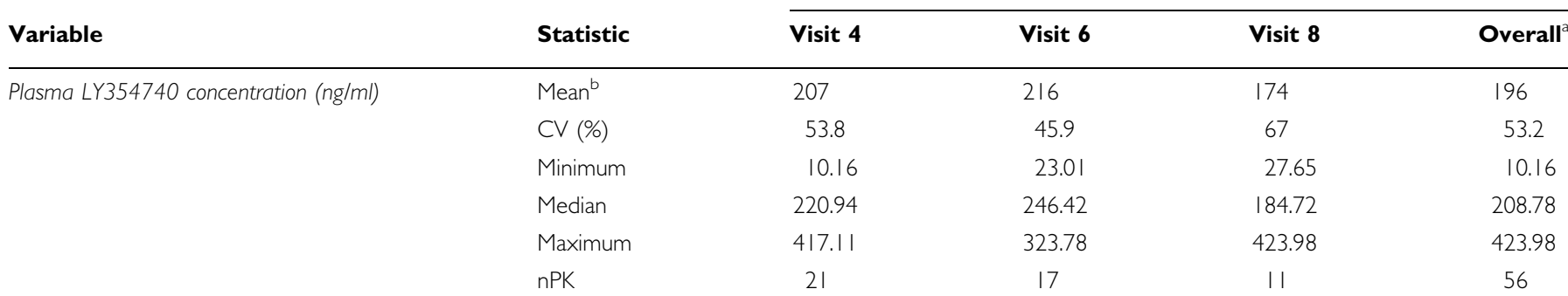

b.i.d., twice daily; CV, coefficient of variation; GAD, generalized anxiety disorder; nPK, number of pharmacokinetic observations.

${ }^{a}$ Quantifiable LY354740 plasma concentrations collected on all visits (visit 4 through visit 8 ) were included in the computation of overall summary statistics.

${ }^{\mathrm{b}}$ Arithmetic mean.

significant differences in several secondary efficacy measures used, such as the HADS, MADRS, or the CGI-S scores for both LY544344 groups. Lack of significant effects on these measures may be due in part to lack of statistical power to detect differences among treatment groups stemming from the diminished sample size. Lack of treatment effect on depressive symptoms may also be due to inclusion criteria limiting depression severity in this sample, as evidenced by the low baseline scores on the MADRS and HADS depression subscale. Alternative explanations may also include therapeutic effects being more apparent in clinician- (eg, HAM-A, CGI-I) as opposed to patient-rated scales (eg, HADS anxiety subscale), or lack of antidepressant effects from mGlu2/3 agonists. The doubleblind design and the benign safety and tolerability profile of LY544344 make it unlikely that awareness of treatment assignment by investigators would have biased efficacy ratings. However, as raters were not blind to patientreported adverse events and because a numerically higher rate of nausea was observed in the LY544344 treatment groups, some degree of unblinding to treatment assignment cannot be ruled out. Limited effects in patient-rated and depressive symptom measures are consistent with LWAY findings (Michelson et al, 2005).

Adverse events were infrequent and mild among the LY544344 and placebo groups, and no serious adverse events were noted. Rates of discontinuation due to adverse events were small, indicating that LY544344 was well tolerated in this population. No clinically significant observations were made regarding changes in vital signs, EKGs, or laboratory assessments, including blood chemistries and hematology. There were no reports of convulsions or other neurobehavioral adverse events. Plasma concentrations of LY354740 were comparable to those previously associated with efficacy in GAD (data on file, Eli Lilly and Co.), confirming the utility of using a dipeptide analog to increase the oral bioavailability (by way of peptide transporters) of otherwise poorly absorbed amino-acid analogs such as LY354740 (Bueno et al, 2005; Rorick-Kehn et al, 2006). The use of the prodrug LY544344 is associated with increased oral bioavailability and decreased variability of active moiety (LY354740) in plasma concentration. This leads to more predictable clinical effects at a given dose and lower manufacturing costs relative to the poorly bioavailable parent drug LY354740.

The efficacy, safety, and tolerability results of this study add to the emerging field of data suggesting the important role of glutamatergic modulation of anxiety and related symptoms (Swanson et al, 2005). Other agents affecting glutamate neurotransmission, such as ketamine, lamotrigine, and riluzole have been available for clinical use for some time (for review, see Zarate et al, 2002). Riluzole, an inhibitor of glutamate release indicated for the treatment of amyotrophic lateral sclerosis, was found efficacious in an open-label GAD trial (Mathew et al, 2005). Of note, LY354740 and its prodrug LY544344 are the first agents to target specific G-protein-linked glutamate receptors that modulate glutamatergic transmission and to be clinically investigated (Swanson et al, 2005). LY354740 is nanomolar 
potent at group II (mGlu2/3) receptors, with no appreciable activity at either group I or III mGlu receptor subtypes or NMDA, AMPA, or kainate ionotropic glutamate receptors. mGlu2/3 receptors are negatively coupled to adenylate cyclase and function to negatively modulate excitatory neurotransmitter output and neuronal excitability (Schoepp, 2001; Schoepp et al, 2003). It is established that the activation of $\mathrm{mGlu} 2 / 3$ receptors leads to reductions in the evoked release of glutamate and concomitant reduction on post-synaptic glutamate-mediated excitations (Schoepp, 2001). Localization studies with mGlu 2 or mGlu2/3 selective antibodies indicate that the mGlu2 receptors are located primarily at presynaptic sites, but they are external to the synaptic junctions (Cartmell and Schoepp, 2000). Thus, mGlu2 receptors likely monitor glutamate, which escapes the synapse upon excessive release of neurotransmitter (eg, high-frequency stimulations) or when uptake processes in glial and/or neurons that clear synaptic glutamate have been compromised. Thus, mGlu2 receptors have an important regulatory role in preventing pathological glutamate excitation from occurring, but play less of a role in physiological excitatory transmission per se. mGlu2 and/or mGlu3 receptors are also present post-synaptically, where they hyperpolarize cells thus decreasing excitability (Tamaru et al, 2001). mGlu3 receptors have been identified on glial cells where their function is less understood (Schoepp, 2001). In brain synapses involved in fear and anxiety responses, mGlu $2 / 3$ receptor agonists have been shown to decrease presynaptic glutamate release and induce postsynaptic hyperpolarization, thus modulating limbic neuronal excitability. The basolateral and central nucleus of the amygdala, medial prefrontal cortex, and the lateral and medial perforant paths of the hippocampus are some of the brain areas where LY354740 can inhibit glutamate-evoked synaptic excitatory responses (Schoepp et al, 2003). These areas have been implicated in modulation of anxiety/fear in animals and humans (Pratt, 1992) and the acquisition and expression of fear/anxiety responses (Davis et al, 1994). In the amygdala, cFOS studies also suggest that LY354740 prevents disinhibition of circuits that drive fear responses by modulating GABAergic transmission (Linden et al, 2004). Modulation of glutamate- or GABA-mediated excitability in limbic circuits by LY354740 likely represents the key mechanism(s) of action that lead to the anxiolytic effects observed with these agents in preclinical anxiety models as well as human studies (Schoepp et al, 2003).

The only known human data with mGlu2/3 agonists to date have been limited to studies with LY354740 or LY544344. In addition to the previously discussed GAD studies, a cholecystokinin tetrapeptide challenge to induce anxiety in healthy volunteers demonstrated equivocal effects of LY544344 $80 \mathrm{mg}$ b.i.d. (Kellner et al, 2005). A double-blind clinical trial with LY354740 was conducted in panic disorder patients. The study found treatment with LY354740 200-400 mg/day no more efficacious than treatment with placebo (Bergink and Westenberg, 2005 and data on file, Eli Lilly and Co.), in opposition to positive LY354740 efficacy findings in a human model of panic anxiety (Schoepp et al, 2003). The reasons for the lack of efficacy in panic disorder are not clear; higher doses or LY354740 exposures that can be achieved with other mGlu2/3 agonists with better bioavailability, such as LY544344, may be needed to treat conditions other than GAD. Few studies have been performed to assess the efficacy of LY354740 or LY544344 in conditions other than GAD, and no LY544344 human studies have been conducted to date in panic or major depressive disorder. Additional clinical studies will be necessary to further outline the spectrum of disorders in which mGlu2/3 agonist agents may be efficacious.

While the mechanism underlying the preclinical findings of convulsions remains unknown, it is unclear to what extent these findings are relevant to humans, as no convulsions were observed in humans receiving LY354740 for more than 1 year, or LY544344 for up to 8 weeks. Convulsions were observed after repeated dosing in an 8-month dog study at LY544344 doses as low as $60 \mathrm{mg} / \mathrm{kg}$, and in 2-year rat and mouse studies at doses as low as 70 and $150 \mathrm{mg} / \mathrm{kg}$, respectively. The observation of convulsions in preclinical species and the potential for increased convulsion risk in humans led to discontinuation of this study. The clinical significance of animal convulsions remains unknown. To establish the potential clinical utility of mGlu2/3 receptor agonist agents, further evaluation of their preclinical and clinical safety and tolerability will be required.

In summary, results of this study appear to confirm previous findings indicating that agonism at mGlu2/3 receptors may be associated with anxiolytic effects in humans. The potential therapeutic role of mGlu2/3 agonists in the treatment of anxiety disorders awaits further preclinical and more definitive clinical evaluation.

\section{ACKNOWLEDGEMENTS}

This work was sponsored and supported by Eli Lilly and Co. This paper was written and assembled for submission with the assistance of Laurane G Mendelsohn, PhD. David Michelson, MD, provided review and useful suggestions of an earlier version of this paper.

Drs Levine, Dunayevich, Landbloom, Erickson, Schoepp, and Tollefson are former Lilly employees when this study was carried out. Drs Dunayevich, Landbloom, Erickson, and Tollefson are currently employed by Orexigen Therapeutics; Dr Schoepp is currently employed by Merck. Dr Tollefson is also a consultant with Eli Lilly and Co. and Volunteer Professor of Clinical Psychiatry at Indiana University.

LMBS investigators: Margot Schmidt, Georg Schonbeck, Austria; Leo Ruelens, Stefaan Geertz, Jan Van Dorpe, Joseph LeJeune, Belgium; Bertrand Baranovsky, Francis Gheysen, Pierre Le Goubey, Marcel Zins-Ritter, Sami Atallah, France; Matteo Balestrieri, Italy; Angel Morinigo, Ignacio Mata Pastor, Spain; Herro F Kraan, Henk Mulder, The Netherlands; Robert C Cook, Alan Middleton, John Ryan, John R Cecil, Andrew S Cowie, Ian Orpen, Ian Parker, Nicholas CR Jones, Mark Blagden, Andrew Matthews, Robin Lal-Sarin, Andrew Smithers, UK.

\section{REFERENCES}

Ballenger JC (2001). Treatment of anxiety disorders to remission. $J$ Clin Psychiatry 62(Suppl 12): 5-9.

Bergink V, Westenberg HG (2005). Metabotropic glutamate II receptor agonists in panic disorder: a double blind clinical trial with LY354740. Int Clin Psychopharmacol 20: 291-293. 
Bueno AB, Collado I, de Dios A, Dominguez C, Martin JA, Martin LM et al (2005). Dipeptides as effective prodrugs of the unnatural amino acid (+)-2-aminobicyclo[3.1.0]hexane-2,6dicarboxylic acid (LY354740), a selective group II metabotropic glutamate receptor agonist. J Med Chem 48: 5305-5320.

Cartmell J, Schoepp DD (2000). Regulation of neurotransmitter release by metabotropic glutamate receptors. J Neurochem 75: 889-907.

Davis M, Rainnie D, Cassell M (1994). Neurotransmission in the rat amygdala related to fear and anxiety. Trends Neurosci 17: 208-214.

Grillon C, Cordova J, Levine LR, Morgan III CA (2003). Anxiolytic effects of a novel group II metabotropic glutamate receptor agonist (LY354740) in the fear-potentiated startle paradigm in humans. Psychopharmacology 168: 446-454.

Guy W (1976). ECDEU Assessment Manual for Psychopharmaco$\log y$ (revised). National Institute of Mental Health, Psychopharmacology Research Branch: Rockville, MD. pp 217-222 and 313-331.

Hamilton M (1959). The assessment of anxiety states by rating. Br J Psychiatry 32: 50-55.

Hedges LV, Olkin I (1985). Statistical Methods for Meta-analysis. Academic Press: San Diego, CA.

Helton DR, Tizzano JP, Monn JA, Schoepp DD, Kallman MJ (1998). Anxiolytic and side-effect profile of LY354740: a potent, highly selective, orally active agonist for group II metabotropic glutamate receptors. J Pharmacol Exp Ther 284: 651-660.

Kellner M, Muhtz C, Stark K, Yassouridis A, Arlt J, Wiedemann K (2005). Effects of a metabotropic glutamate $(2 / 3)$ receptor agonist (LY544344/LY354740) on panic anxiety induced by cholecystokinin tetrapeptide in healthy humans: preliminary results. Psychopharmacology (Berlin) 179: 310-315.

Linden AM, Greene SJ, Bergeron M, Schoepp DD (2004). Anxiolytic activity of the MGLU2/3 receptor agonist LY354740 on the elevated plus maze is associated with the suppression of stress-induced c-Fos in the hippocampus and increases in c-Fos induction in several other stress-sensitive brain regions. Neuropsychopharmacology 29: 502-513.

Linden AM, Shannon H, Baez M, Yu J, Koester A, Schoepp DD (2005). Anxiolytic activity of the mGlu2/3 receptor agonist LY354740 in the elevated plus maze test is disrupted in metabotropic glutamate receptor 2 and 3 knock-out mice. Psychopharmacology 179: 284-291.

Lipman RS (1982). Differentiating anxiety and depression in anxiety disorders: use of rating scales. Psychopharmacol Bull 18: 69-77.

Mathew SJ, Amiel J, Coplan J, Fitterling H, Sackeim H, Gorman J (2005). Open-label trial of riluzole in generalized anxiety disorder. Am J Psychiatry 162: 2379-2381.

Michelson D, Levine LR, Dellva MA, Mesters P, Schoepp DD, Dunayevich E et al (2005). Clinical studies with mGlu2/3 receptor agonists: LY354740 compared with placebo in patients with generalized anxiety disorder. Neuropharmacology 49(S1): 84-257.

Monn JA, Valli MJ, Massey SM, Wright RA, Salhoff CR, Johnson BG et al (1997). Design, synthesis, and pharmacological characterization of (+)-2-aminobicyclo[3.1.0]hexane-2,6-dicarboxylic acid (LY354740): a potent, selective, and orally active group 2 metabotropic glutamate receptor agonist possessing anticonvulsant and anxiolytic properties. J Med Chem 40: 528-537.

Montgomery SA, Asberg M (1979). A new depression scale designed to be sensitive to change. Br J Psychiatry 134: 382-389.
Moore HK, Mundt JC, Modell JG, Rodrigues HE, DeBrota DJ, Jefferson JJ et al (2006). An examination of 26,168 Hamilton Depression Rating Scale scores administered via interactive voice response across 17 randomized clinical trials. J Clin Psychopharmacol 26: 321-324.

Pin JP, Duvoisin R (1995). The metabotropic glutamate receptors: structure and functions. Neuropharmacology 34: 1-26.

Pratt JA (1992). The neuroanatomical basis of anxiety. Pharmacol Ther 55: 149-181.

Raskin A, Schulterbrandt J, Reating N, McKeon JJ (1969). Replication of factors of psychopathology in interview, ward behavior and self-report ratings of hospitalized depressives. J Nerv Ment Dis 148: 87-98.

Rorick-Kehn LM, Perkins EJ, Knitowski KM, Hart JC, Johnson BG, Schoepp DD et al (2006). Improved bioavailability of the mGlu2/ 3 receptor agonist LY354740 using a prodrug strategy: in vivo pharmacology of LY544344. J Pharmacol Exp Ther 316: 905-913.

Schoepp DD (2001). Unveiling the functions of presynaptic metabotropic glutamate receptors in the central nervous system. J Pharmacol Exp Ther 299: 12-20.

Schoepp DD, Johnson BG, Wright RA, Salhoff CR, Mayne NG, Wu S et al (1997). LY354740 is a potent and highly selective group II metabotropic glutamate receptor agonist in cells expressing human glutamate receptors. Neuropharmacology 36: $1-11$.

Schoepp DD, Wright RA, Levine LR, Gaydos B, Potter WZ (2003). LY354740, an mGlu2/3 receptor agonist as a novel approach to treat anxiety/stress. Stress 6: 189-197.

Sheehan DV, Lecrubier Y, Sheehan KH, Amorim P, Janavs J, Weiller E et al (1998). The Mini-International Neuropsychiatric Interview (MINI): the development and validation of a structured diagnostic psychiatric interview for DSM-IV and ICD-10. J Clin Psychiatry 9(Suppl 20): 22-33.

Shekhar A, Keim SR (2000). LY354740, a potent group II metabotropic glutamate receptor agonist prevents lactate-induced panic-like response in panic-prone rats. Neuropharmacology 39: 1139-1146.

Spooren WP, Schoeffter P, Gasparini F, Kuhn R, Gentsch C (2002). Pharmacological and endocrinological characterisation of stressinduced hyperthermia in singly housed mice using classical and candidate anxiolytics (LY314582, MPEP and NKP608). Eur J Pharmacol 435: 161-170.

Swanson CJ, Bures M, Johnson MP, Linden AM, Monn JA, Schoepp DD (2005). Metabotropic glutamate receptors as novel targets for anxiety and stress disorders. Nat Rev Drug Discov 4: 131-144.

Tamaru Y, Nomura S, Mizuno N, Shigemoto R (2001). Distribution of metabotropic glutamate receptor mGluR3 in the mouse CNS: differential location relative to pre- and post-synaptic sites. Neuroscience 106: 481-503.

Tizzano JP, Griffey KI, Schoepp DD (2002). The anxiolytic action of $\mathrm{mGlu} 2 / 3$ receptor agonist, LY354740, in the fear-potentiated startle model in rats is mechanistically distinct from diazepam. Pharmacol Biochem Behav 73: 367-374.

Walker DL, Rattiner LM, Davis M (2002). Group II metabotropic glutamate receptors within the amygdala regulate fear as assessed with potentiated startle in rats. Behav Neurosci 116: 1075-1083

Zarate CA, Quiroz J, Payne J (2002). Modulators of the glutamatergic system: implications for the development of improved therapeutics in mood disorders. Psychopharmacol Bull 36: 35-83.

Zigmond AS, Snaith RP (1983). The hospital anxiety and depression scale. Acta Psychiatr Scand 67: 361-370. 